\title{
Muon anomalous magnetic moment and positron excess at AMS-02 in a gauged horizontal symmetric model
}

\author{
Gaurav Tomar ${ }^{a, b}$ and Subhendra Mohanty ${ }^{a}$ \\ a Physical Research Laboratory, \\ Ahmedabad 380009, India \\ ${ }^{b}$ Indian Institute of Technology, \\ Gandhinagar 382424, India \\ E-mail: tomar@prl.res.in, mohanty@prl.res.in
}

ABSTRACT: We studied an extension of the standard model with a fourth generation of fermions to explain the discrepancy in the muon $(g-2)$ and explain the positron excess seen in the AMS-02 experiment. We introduce a gauged $\mathrm{SU}(2)_{\mathrm{HV}}$ horizontal symmetry between the muon and the 4 th generation lepton families. The 4 th generation right-handed neutrino is identified as the dark matter with mass $\sim 700 \mathrm{GeV}$. The dark matter annihilates only to $\left(\mu^{+} \mu^{-}\right)$and $\left(\nu_{\mu}^{c} \nu_{\mu}\right)$ states via $\mathrm{SU}(2)_{\mathrm{HV}}$ gauge boson. The $\mathrm{SU}(2)_{\mathrm{HV}}$ gauge boson with mass $\sim 1.4 \mathrm{TeV}$ gives an adequate contribution to the $(g-2)$ of muon and fulfill the experimental constraint from BNL measurement. The higgs production constraints from 4th generation fermions is evaded by extending the higgs sector.

Keywords: Beyond Standard Model, Cosmology of Theories beyond the SM

ARXIV EPRINT: 1403.6301 


\section{Contents}

1 Introduction 1

2 Model 3

3 Dark matter phenomenology 5

3.1 Relic density 6

$\begin{array}{lll}3.2 & \text { Comparison with AMS-02 and PAMELA data } & 7\end{array}$

4 Muon magnetic moment $\quad 9$

$\begin{array}{llr}5 & \text { Result and discussion } & 12\end{array}$

\section{Introduction}

There exist two interesting experimental signals namely the muon $(g-2)$, measured at BNL $[1,2]$ and the excess of positrons measured by AMS-02 [20, 21], which may have a common beyond standard model (SM) explanation.

There is a discrepancy at $3.6 \sigma$ level between the experimental measurement $[1,2]$ and the SM prediction [3-9] of muon anomalous magnetic moment,

$$
\Delta a_{\mu} \equiv a_{\mu}^{\mathrm{Exp}}-a_{\mu}^{\mathrm{SM}}=(28.7 \pm 8.0) \times 10^{-10}
$$

where $a_{\mu}$ is the anomalous magnetic moment in the unit of $e / 2 m_{\mu}$. In the standard model, contribution of $W$ boson to the muon anomalous magnetic magnetic moment goes as $a_{\mu}^{W} \propto m_{\mu}^{2} / M_{W}^{2}$ and we have $a_{\mu}^{\mathrm{SM}}=19.48 \times 10^{-10}[10]$.

In minimal supersymmetric standard model (MSSM) [11, 12], we get contributions to muon $(g-2)$ from neutralino-smuon and chargino-sneutrino loops. In all MSSM diagrams there still exist a $m_{\mu}$ suppression in $(g-2)$, arising from the following cases: (a) in case of bino in the loop, the mixing between the left and right handed smuons is $\propto m_{\mu}$ (b) in case of wino-higgsino or bino-higgsino in the loop, the higgsino coupling with smuon is $\propto y_{\mu}$, so there is a $m_{\mu}$ suppression (c) in the case of chargino-sneutrino in the loop, the higgsinomuon coupling is $\propto y_{\mu}$, which again gives rise to $m_{\mu}$ suppression. Therefor in MSSM $a_{\mu}^{\mathrm{MSSM}} \propto m_{\mu}^{2} / M_{\mathrm{SUSY}}^{2}$, where $M_{\mathrm{SUSY}}$ is proportional to the mass of the SUSY particle in the loop.

One can evade the muon mass suppression in $(g-2)$ with a horizontal gauge symmetry. In [13] a horizontal $\mathrm{U}(1)_{L_{\mu}-L_{\tau}}$ symmetry was used in which muon $(g-2)$ is proportional to $m_{\tau}$ and $a_{\mu} \propto m_{\mu} m_{\tau} / m_{Z^{\prime}}^{2}$, where $L_{\mu}-L_{\tau}$ gauge boson mass $m_{Z^{\prime}} \propto 100 \mathrm{GeV}$ gives the required $a_{\mu}$. A model independent analysis of the beyond SM particles which can give a contribution to $a_{\mu}$ is studied in [14]. The SM extension needed to explain muon $(g-2)$ has 
also been related to dark matter $[15,16]$ and the implication of this new physics in LHC searches has been studied [17]. An explanation of $(g-2)$ from the 4th generation leptons has also been given in $[18,19]$.

The second experimental signal, which we address in this paper is the excess of positron over cosmic-ray background, which has been observed by AMS-02 experiment [20] upto energy $\sim 425 \mathrm{GeV}[21]$. An analysis of AMS-02 data suggests that a dark matter (DM) annihilation interpretation would imply that the annihilation final states are either $\mu$ or $\tau[23,24]$. The dark matter annihilation into $e^{ \pm}$pairs would give a peak in positron signal, which is not seen in the positron spectrum. The branching ratio of $\tau$ decay to $e$ is only $17 \%$ compared to $\mu$, which makes $\mu$ as the preferred source as origin of high energy positrons. The AMS-02 experiment does not observe an excess, beyond the cosmic-ray background, in the antiproton flux [25, 26], indicating a leptophilic dark matter [27, 28, 33].

In this paper, we introduce a 4 th generation of fermions and a $\mathrm{SU}(2)_{\mathrm{HV}}$ vector gauge symmetry between the 4 th generation leptons and the muon families. In our model, the muon $(g-2)$ has a contribution from the 4 th generation charged lepton $\mu^{\prime}$, and the $\mathrm{SU}(2)_{\mathrm{HV}}$ gauge boson $\theta^{+}$,

$$
\Delta a_{\mu} \propto \frac{m_{\mu} m_{\mu^{\prime}}}{M_{\theta^{+}}^{2}}
$$

and from the neutral higgs scalars $(h, A)$,

$$
\Delta a_{\mu} \propto \frac{m_{\mu}}{m_{\mu^{\prime}}}
$$

and from the charged higgs $H^{ \pm}$the contribution is,

$$
\Delta a_{\mu} \propto-\frac{m_{\mu} m_{\nu_{\mu^{\prime}}}}{m_{H^{ \pm}}^{2}}
$$

In all these cases, there is no quadratic suppression $\propto m_{\mu}^{2}$ because of the horizontal symmetry. By choosing parameters of the model without any fine tunning, we can obtain the required number $\Delta a_{\mu}=2.87 \times 10^{-9}$ within $1 \sigma$.

In this model, the 4 th generation right-handed neutrino $\nu_{\mu^{\prime} R}$, is identified as dark matter. The dark matter annihilates to the standard model particles through the $\mathrm{SU}(2)_{\mathrm{HV}}$ gauge boson $\theta_{3}$ and with the only final states being $\left(\mu^{+} \mu^{-}\right)$and $\left(\nu_{\mu}^{c} \nu_{\mu}\right)$. The stability of DM is maintained by taking the 4th generation charged lepton to be heavier than DM. To explain the AMS-02 signal [20,21], one needs a cross-section (CS), $\sigma v_{\chi \chi \rightarrow \mu^{+} \mu^{-}}=$ $2.33 \times 10^{-25} \mathrm{~cm}^{3} / \mathrm{sec}$, which is larger than the CS, $\sigma v_{\chi \chi \rightarrow S M} \sim 3 \times 10^{-26} \mathrm{~cm}^{3} / \mathrm{sec}$, required to get the correct thermal relic density $\Omega h^{2}=0.1199 \pm 0.0027[29,30]$. In our model, the enhancement of annihilation CS of DM in the galaxy is achieved by the resonant enhancement mechanism [31-33], which we attain by taking $M_{\theta_{3}} \simeq 2 m_{\chi}$.

This paper is organized as follows: in section 2, we describe the model. In section 3 we discuss the dark matter phenomenology and in section 4 , we compute the $(g-2)$ contributions from this model and then give our conclusion in section 5 . 


\begin{tabular}{|c|c|}
\hline Particles & $G_{\mathrm{STD}} \times \mathrm{SU}(2)_{\mathrm{HV}}$ quantum numbers \\
\hline$\psi_{e L i} \equiv\left(\nu_{e}, e\right)$ & $(1,2,-1,1)$ \\
\hline$\Psi_{L i \alpha} \equiv\left(\psi_{\mu}, \psi_{\mu^{\prime}}\right)$ & $(1,2,-1,2)$ \\
\hline$\psi_{\tau L i} \equiv\left(\nu_{\tau}, \tau\right)$ & $(1,2,-1,1)$ \\
\hline$E_{R \alpha} \equiv\left(\mu_{R}, \mu_{R}^{\prime}\right)$ & $(1,1,-2,2)$ \\
\hline$N_{R \alpha} \equiv\left(\nu_{\mu R}, \nu_{\mu^{\prime} R}\right)$ & $(1,1,0,2)$ \\
\hline$e_{R}, \tau_{R}$ & $(1,1,-2,1)$ \\
\hline$\nu_{e R}, \nu_{\tau R}$ & $(1,1,0,1)$ \\
\hline$\phi_{i}$ & $(1,2,1,1)$ \\
\hline$\eta_{i \alpha}^{\beta}$ & $(1,2,1,3)$ \\
\hline$\chi_{\alpha}$ & $(1,1,0,2)$ \\
\hline
\end{tabular}

Table 1. Representation of the various fields in the model under the gauge group $G_{\mathrm{STD}} \times \mathrm{SU}(2)_{\mathrm{HV}}$.

\section{Model}

In addition to the three generations of quarks and leptons, we introduce the 4th generation of quarks $\left(c^{\prime}, s^{\prime}\right)$ and leptons $\left(\nu_{\mu}^{\prime}, \mu^{\prime}\right)$ (of both chiralities) in the standard model. We also add three right-handed neutrinos and extend the gauge group of SM by horizontal symmetry denoted by $\mathrm{SU}(2)_{\mathrm{HV}}$, between the 4th generation lepton and muon families. Addition of three right-handed neutrinos ensures that the model is free from SU(2) Witten anomaly [34]. We assume that the quarks of all four generations and the leptons of $e$ and $\tau$ families are singlet of $\mathrm{SU}(2)_{\mathrm{HV}}$ to evade the constraints from flavour changing processes. The $\mathrm{SU}(2)_{\mathrm{HV}}$ symmetry can be extended to $e$ and $\tau$ families by choosing suitable discrete symmetries, however in this paper we have taken $e$ and $\tau$ families to be singlet of $\mathrm{SU}(2)_{\mathrm{HV}}$ for simplicity and discuss the most economical model, which can explain muon $(g-2)$ and AMS-02 positron excess at the same time.

We denote the left-handed muon and 4th generation lepton families by $\Psi_{L i \alpha}$ and their right-handed charged and neutral counterparts by $E_{R \alpha}$ and $N_{R \alpha}$ respectively (here $i$ and $\alpha$ are the $\mathrm{SU}(2)_{L}$ and $\mathrm{SU}(2)_{\mathrm{HV}}$ indices respectively and run through the values 1 and 2 ). The left-handed electron and tau doublets are denoted by $\psi_{e L i}$ and $\psi_{\tau L i}$ and their right-handed counterparts by $e_{R}$ and $\tau_{R}$ respectively. The gauge fields of $\mathrm{SU}(2)_{L} \times \mathrm{U}(1)_{Y} \times \mathrm{SU}(2)_{\mathrm{HV}}$ groups are denoted by $A_{\mu}^{a}, B_{\mu}$ and $\theta_{\mu}^{a}(a=1,2,3)$ with gauge couplings $g, g^{\prime}$ and $g_{H}$ respectively.

The leptons transformations under the gauge group, $\mathrm{SU}(3)_{c} \times \mathrm{SU}(2)_{L} \times \mathrm{U}(1)_{Y} \times$ $\mathrm{SU}(2)_{\mathrm{HV}} \equiv G_{\mathrm{STD}} \times \mathrm{SU}(2)_{\mathrm{HV}}$ are shown in table 1 . From the assigned quantum numbers, it is clear that the $\mathrm{SU}(2)_{\mathrm{HV}}$ gauge bosons connect only the leptons pairs, $\psi_{\mu_{L}} \leftrightarrow \psi_{\mu_{L}^{\prime}}$ and $\left(\mu_{R}, \nu_{\mu R}\right) \leftrightarrow\left(\mu_{R}^{\prime}, \nu_{\mu R}^{\prime}\right)$. This assignment prevents the flavour changing process like $\mu \rightarrow e \gamma$ for which there are stringent bounds, and also ensures the contribution of heavy lepton $\mu^{\prime}$ to the muon $(g-2)$ as shown in figure 4 . In our $G_{\mathrm{STD}} \times \mathrm{SU}(2)_{\mathrm{HV}}$ model, the 
gauge couplings of the muon and 4th generation lepton families are,

$$
\begin{aligned}
\mathcal{L}_{\psi}= & i \bar{\Psi}_{L i \alpha} \gamma^{\mu}\left(\partial_{\mu}-\frac{i}{2} g \tau \cdot A_{\mu}+i g^{\prime} B_{\mu}-\frac{i}{2} g_{H} \tau \cdot \theta_{\mu}\right)_{i j ; \alpha \beta} \Psi_{L j \beta} \\
& +i \bar{E}_{R \alpha} \gamma^{\mu}\left(\partial_{\mu}+i 2 g^{\prime} B_{\mu}-\frac{i}{2} g_{H} \tau \cdot \theta_{\mu}\right)_{\alpha \beta} E_{R \beta}+i \bar{N}_{R \alpha} \gamma^{\mu}\left(\partial_{\mu}-\frac{i}{2} g_{H} \tau \cdot \theta_{\mu}\right)_{\alpha \beta} N_{R \beta}
\end{aligned}
$$

The "neutral-current" of $\mathrm{SU}(2)_{\mathrm{HV}}$ contributes to the annihilation process, $\left(\nu_{\mu^{\prime}} \nu_{\mu^{\prime}}\right) \rightarrow \theta_{3}^{*} \rightarrow$ $\left(\mu^{+} \mu^{-}\right),\left(\nu_{\mu}^{c} \nu_{\mu}\right)$, which is relevant for the AMS-02 and relic density calculations. The "charge-changing" vertex $\mu \mu^{\prime} \theta^{+}$, contributes to the $(g-2)$ of the muon.

To evade the bounds on the 4th generation from the higgs production at LHC, we extend the higgs sector (in addition to $\phi_{i}$ ) by a scalar $\eta_{i \alpha}^{\beta}$, which is a doublet under $\operatorname{SU}(2)$ and triplet under $\mathrm{SU}(2)_{\mathrm{HV}}$. As a $\mathrm{SU}(2)$ doublet $\eta_{i \alpha}^{\beta}$ evades 4 th generation bounds from the overproduction of higgs in the same way as [35, 36], in that the $125 \mathrm{GeV}$ mass eigenstate is predominantly $\eta$ which has no Yukawa couplings with the quarks. As $\eta_{i \alpha}^{\beta}$ is a triplet under $\mathrm{SU}(2)_{\mathrm{HV}}$, its Yukawa couplings with the muon and 4th generation lepton families split the masses of the muon and 4th generation leptons. We also introduce a $\mathrm{SU}(2)_{\mathrm{HV}}$ doublet $\chi_{\alpha}$, which generates masses for $\mathrm{SU}(2)_{\mathrm{HV}}$ gauge bosons. The quantum numbers of the scalars are shown in table 1 . The general potential of this set of scalars $\left(\phi_{i}, \eta_{i \alpha}^{\beta}, \chi_{\alpha}\right)$ is given in [37]. Following [37], we take the vacuum expectation values (vevs) of scalars as,

$$
\begin{aligned}
\left\langle\phi_{i}\right\rangle & =\langle\phi\rangle \delta_{i 2} \\
\left\langle\eta_{i \alpha}^{\beta}\right\rangle & =\langle\eta\rangle \delta_{i 2}\left(\delta_{\alpha 1} \delta^{\beta 1}-\delta_{\alpha 2} \delta^{\beta 2}\right) \\
|\langle\chi\rangle|^{2} & =\left|\left\langle\chi_{1}\right\rangle\right|^{2}+\left|\left\langle\chi_{2}\right\rangle\right|^{2}
\end{aligned}
$$

where $\left\langle\phi_{i}\right\rangle$ breaks $\mathrm{SU}(2)_{L},\left\langle\chi_{\alpha}\right\rangle$ breaks $\mathrm{SU}(2)_{\mathrm{HV}}$ and $\left\langle\eta_{i \alpha}^{\beta}\right\rangle$ breaks both $\mathrm{SU}(2)_{L}$ and $\mathrm{SU}(2)_{\mathrm{HV}}$ and generate the $\mathrm{TeV}$ scale masses for $\mathrm{SU}(2)_{\mathrm{HV}}$ gauge bosons. The mass eigenstates of the scalars will be a linear combination of $\phi_{i}, \eta_{i \alpha}^{\beta}$ and $\chi_{\alpha}$. We shall assume that the lowest mass eigenstate $h_{1}$ with the mass $\sim 125 \mathrm{GeV}$ is primarily constituted by $\eta_{i \alpha}^{\beta}$. We shall also assume that the parameters of the higgs potential [37] are tuned such that mixing between $h_{1}$ and $\phi_{i}$ is small,

$$
\left\langle h_{1} \mid \phi_{i}\right\rangle \simeq 10^{-2}
$$

The Yukawa couplings of 4 th generation quarks are only with $\phi_{i}$, therefore the $125 \mathrm{GeV}$ Higgs will have very small contribution from the 4th generation quarks loop.

The gauge couplings of the scalar fields $\phi_{i}, \eta_{i \alpha}^{\beta}$ and $\chi_{\alpha}$ are given by the Lagrangian,

$$
\begin{aligned}
\mathcal{L}_{s}= & \left|\left(\partial_{\mu}-\frac{i}{2} g \tau \cdot A_{\mu}-i g^{\prime} B_{\mu}\right) \phi\right|^{2}+\left|\left(\partial_{\mu}-\frac{i}{2} g \tau \cdot A_{\mu}-i g^{\prime} B_{\mu}-i g_{H} T \cdot \theta_{\mu}\right) \eta\right|^{2} \\
& +\left|\left(\partial_{\mu}-\frac{i}{2} g_{H} \tau \cdot \theta\right) \chi\right|^{2}
\end{aligned}
$$

where $\tau_{a} / 2(a=1,2,3)$ are $2 \times 2$ matrix representation for the generators of $\mathrm{SU}(2)$ and $T_{a}(a=1,2,3)$ are $3 \times 3$ matrix representation for the generators of $\mathrm{SU}(2)$. After expanding 
$\mathcal{L}_{s}$ around the vevs defined in eq. (2.2), the masses of gauge bosons come,

$$
\begin{aligned}
M_{W}^{2} & =\frac{g^{2}}{2}\left(2\langle\eta\rangle^{2}+\langle\phi\rangle^{2}\right), & M_{Z}^{2} & =\frac{g^{2}}{2} \sec ^{2} \theta_{W}\left(2\langle\eta\rangle^{2}+\langle\phi\rangle^{2}\right), \\
M_{\theta^{+}}^{2} & =g_{H}^{2}\left(4\langle\eta\rangle^{2}+\frac{1}{2}\langle\chi\rangle^{2}\right), & M_{A}^{2}=0, & =\frac{1}{2} g_{H}^{2}\langle\chi\rangle^{2}
\end{aligned}
$$

we tune the parameters in the potential such that the vevs of scalars are,

$$
\begin{aligned}
2\langle\eta\rangle^{2}+\langle\phi\rangle^{2} & =(174 \mathrm{GeV})^{2} \\
\langle\chi\rangle & =22.7 \mathrm{TeV}
\end{aligned}
$$

for the generation of large masses for 4 th generation leptons $\mu^{\prime}, \nu_{\mu^{\prime}}$ and $\mathrm{SU}(2)_{\mathrm{HV}}$ gauge bosons $\theta^{+}, \theta_{3}$. The Yukawa couplings of the leptons are given by,

$$
\begin{aligned}
\mathcal{L}_{Y}= & -h_{1} \bar{\psi}_{e L i} \phi_{i} e_{R}-\tilde{h}_{1} \epsilon_{i j} \bar{\psi}_{e L i} \phi^{j} \nu_{e R}-h_{2} \bar{\Psi}_{L i \alpha} \phi_{i} E_{R \alpha}-\tilde{h}_{2} \epsilon_{i j} \bar{\Psi}_{L i \alpha} \phi^{j} N_{R \alpha}-k_{2} \bar{\Psi}_{L i \alpha} \eta_{i \alpha}^{\beta} E_{R \beta} \\
& -\tilde{k}_{2} \epsilon_{i j} \bar{\Psi}_{L i \alpha} \eta_{\alpha}^{j \beta} N_{R \beta}-h_{3} \bar{\psi}_{\tau L i} \phi_{i} \tau_{R}-\tilde{h}_{3} \epsilon_{i j} \bar{\psi}_{\tau L i} \phi^{j} \nu_{\tau R}+\text { h.c. }
\end{aligned}
$$

after corresponding scalars take their vevs as defined in eq. (2.2), we obtain

$$
\begin{aligned}
\mathcal{L}_{Y}= & -h_{1} \bar{\psi}_{e L 2}\langle\phi\rangle e_{R}-\tilde{h}_{1} \bar{\psi}_{e L 1}\langle\phi\rangle \nu_{e R}-\bar{\Psi}_{L 2 \alpha}\left[h_{2}\langle\phi\rangle+k_{2}\langle\eta\rangle\left(\delta_{\alpha 1}-\delta_{\alpha 2}\right)\right] E_{R \alpha} \\
& -\bar{\Psi}_{L 1 \alpha}\left[\tilde{h}_{2}\langle\phi\rangle+\tilde{k}_{2}\langle\eta\rangle\left(\delta_{\alpha 1}-\delta_{\alpha 2}\right)\right] N_{R \alpha}-h_{3} \bar{\psi}_{\tau L 2}\langle\phi\rangle \tau_{R}-\tilde{h}_{3} \bar{\psi}_{\tau L 1}\langle\phi\rangle \nu_{\tau R} \\
& -h_{1} \bar{\psi}_{e L i} \phi_{i}^{\prime} e_{R}-\tilde{h}_{1} \epsilon_{i j} \bar{\psi}_{e L i} \phi^{\prime j} \nu_{e R}-\bar{\Psi}_{L i \alpha}\left[h_{2} \phi_{i}^{\prime} \delta_{\alpha}^{\beta}+k_{2} \eta_{i \alpha}^{\prime \beta}\right] E_{R \beta} \\
& -\bar{\Psi}_{L i \alpha}\left[\tilde{h}_{2} \epsilon_{i j} \phi^{\prime j} \delta_{\alpha}^{\beta}+\tilde{k}_{2} \epsilon_{i j} \eta_{\alpha}^{\prime j \beta}\right] N_{R \beta}-h_{3} \bar{\psi}_{\tau L i} \phi_{i}^{\prime} \tau_{R}-\tilde{h}_{3} \epsilon_{i j} \bar{\psi}_{\tau L i} \phi^{\prime j} \nu_{\tau R}+\text { h.c. }
\end{aligned}
$$

where $\phi_{i}^{\prime}$ and $\eta_{i \alpha}^{\prime \beta}$ are the shifted fields. From eq. (2.8), we see that the muon and 4th generation leptons masses get split and are given by,

$$
\begin{aligned}
& m_{e}=h_{1}\langle\phi\rangle, \quad m_{\tau}=h_{3}\langle\phi\rangle, \quad m_{\nu_{e}}=\tilde{h}_{1}\langle\phi\rangle, \quad m_{\nu_{\tau}}=\tilde{h}_{3}\langle\phi\rangle \\
& m_{\mu}=h_{2}\langle\phi\rangle+k_{2}\langle\eta\rangle, \quad m_{\nu_{\mu}}=\tilde{h}_{2}\langle\phi\rangle+\tilde{k}_{2}\langle\eta\rangle, \\
& m_{\mu^{\prime}}=h_{2}\langle\phi\rangle-k_{2}\langle\eta\rangle, \quad m_{\nu_{\mu^{\prime}}}=\tilde{h}_{2}\langle\phi\rangle-\tilde{k}_{2}\langle\eta\rangle
\end{aligned}
$$

Thus by choosing the suitable values of Yukawas, the required leptons masses can be generated.

\section{Dark matter phenomenology}

In our model, we identify the 4 th generation right-handed neutral lepton $\left(\nu_{\mu_{R}}^{\prime} \equiv \chi\right)$ as the dark matter, which is used to fit AMS-02 data [20, 21]. The only possible channels for $\mathrm{DM}$ annihilation are into $\left(\mu^{+} \mu^{-}\right)$and $\left(\nu_{\mu}^{c} \nu_{\mu}\right)$ pairs (figure 1$)$. In this scenario for getting the correct relic density, we use the Breit-Wigner resonant enhancement [31-33] and take $M_{\theta_{3}} \simeq 2 m_{\chi}$. The annihilation CS can be tuned to be $\sim 10^{-26} \mathrm{~cm}^{3} \mathrm{~s}^{-1}$ with the resonant enhancement, which gives the observed relic density. In principle the dark matter can decay into the light leptons via $\mathrm{SU}(2)_{\mathrm{HV}}$ gauge boson $\theta^{+}$and scalar $\eta_{i \alpha}^{\beta}$, but by taking the mass of 4 th generation charged leptons $\mu^{\prime}$ larger than $\chi$, the stability of dark matter can be ensured. 


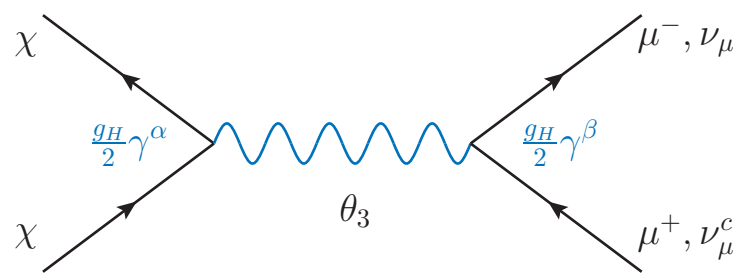

Figure 1. Feynman diagram of dark matter annihilation with corresponding vertex factor.

\subsection{Relic density}

The dark matter annihilation channels into standard model particles are, $\chi \chi \rightarrow \theta_{3}^{*} \rightarrow$ $\mu^{+} \mu^{-}, \nu_{\mu}^{c} \nu_{\mu}$. The annihilation rate of dark matter $\sigma v$, for a single channel, in the limit of massless leptons, is given by

$$
\sigma v=\frac{1}{16 \pi} \frac{g_{H}^{4} m_{\chi}^{2}}{\left(s-M_{\theta_{3}}^{2}\right)^{2}+\Gamma_{\theta_{3}}^{2} M_{\theta_{3}}^{2}}
$$

where $g_{H}$ is the horizontal gauge boson coupling, $m_{\chi}$ the dark matter mass, $M_{\theta_{3}}$ and $\Gamma_{\theta_{3}}$ are the mass and the decay width of $\mathrm{SU}(2)_{\mathrm{HV}}$ gauge boson respectively. Since both of the final states $\left(\nu_{\mu}, \mu\right)$ contribute in the relic density, the cross-section of eq. (3.1) is multiplied by a factor of 2 for relic density computation. The contributions to the decay width of $\theta_{3}$ comes from the decay modes, $\theta_{3} \rightarrow \mu^{+} \mu^{-}, \nu_{\mu}^{c} \nu_{\mu}$. The total decay width is given by,

$$
\Gamma_{\theta_{3}}=\frac{2 g_{H}^{2}}{48 \pi} M_{\theta_{3}}
$$

In the non-relativistic limit, $s=4 m_{\chi}^{2}\left(1+v^{2} / 4\right)$, then by taking into account the factor of 2, eq. (3.1) simplifies as,

$$
\sigma v=\frac{2}{256 \pi m_{\chi}^{2}} \frac{g_{H}^{4}}{\left(\delta+v^{2} / 4\right)^{2}+\gamma^{2}}
$$

where $\delta$ and $\gamma$ are defined as $M_{\theta_{3}}^{2} \equiv 4 m_{\chi}^{2}(1-\delta)$, and $\gamma^{2} \equiv \Gamma_{\theta_{3}}^{2}(1-\delta) / 4 m_{\chi}^{2}$. If $\delta$ and $\gamma$ are larger than $v^{2} \simeq\left(T / M_{\chi}\right)^{2}$, the usual freeze-out takes place, on the other hand if $\delta$ and $\gamma$ are chosen smaller than $v^{2}$ then there is a resonant enhancement of the annihilation CS and a late time freeze-out. We choose $\delta \sim 10^{-3}$ and $\gamma \sim 10^{-4}$, so that we have a resonant annihilation of dark matter. The thermal average of annihilation rate is given as [31-33],

$$
\langle\sigma v\rangle(x)=\frac{1}{n_{\mathrm{EQ}}^{2}} \frac{m_{\chi}}{64 \pi^{4} x} \int_{4 m_{\chi}^{2}}^{\infty} \hat{\sigma}(s) \sqrt{s} K_{1}\left(\frac{x \sqrt{s}}{m_{\chi}}\right) d s
$$

where,

$$
\begin{aligned}
n_{\mathrm{EQ}}^{2} & =\frac{g_{i}}{2 \pi^{2}} \frac{m_{\chi}^{3}}{x} K_{2}(x) \\
\hat{\sigma}(s) & =2 g_{i}^{2} m_{\chi} \sqrt{s-4 m_{\chi}^{2}} \sigma v
\end{aligned}
$$


and where $x \equiv m_{\chi} / T ; K_{1}(x), K_{2}(x)$ represent the modified Bessel functions of second type and $g_{i}$ is the internal degree of freedom of DM particle. Using eq. (3.3), eq. (3.5) and eq. (3.6) in eq. (3.4), it can be written as,

$$
\langle\sigma v\rangle(x)=\frac{g_{H}^{4}}{512 m_{\chi}^{2}} \frac{x^{3 / 2}}{\pi^{3 / 2}} \int_{0}^{\infty} \frac{\sqrt{z} \operatorname{Exp}[-x z / 4]}{(\delta+z / 4)^{2}+\gamma^{2}} d z
$$

where $z \equiv v^{2}$. We solve the Boltzmann equation for $Y_{\chi}=n_{\chi} / s$,

$$
\frac{d Y_{\chi}}{d x}=-\frac{\lambda(x)}{x^{2}}\left(Y_{\chi}^{2}(x)-Y_{\chi \mathrm{eq}}^{2}(x)\right)
$$

where

$$
\lambda(x) \equiv\left(\frac{\pi}{45}\right)^{1 / 2} m_{\chi} M_{\mathrm{Pl}}\left(\frac{g_{* s}}{\sqrt{g_{*}}}\right)\langle\sigma v\rangle(x)
$$

and where $g_{*}$ and $g_{* s}$ are the effective degrees of freedom of the energy density and entropy density respectively, with $\langle\sigma v\rangle$ given in eq. (3.7). We can write the $Y_{\chi}\left(x_{0}\right)$ at the present epoch as,

$$
\frac{1}{Y_{\chi}\left(x_{0}\right)}=\frac{1}{Y_{\chi}\left(x_{f}\right)}+\int_{x_{f}}^{x_{s}} d x \frac{\lambda(x)}{x^{2}}
$$

where the freeze-out $x_{f}$ is obtained by solving $n_{\chi}\left(x_{f}\right)\langle\sigma v\rangle=H\left(x_{f}\right)$. We find that $x_{f} \sim 30$ and the relic density of $\chi$ is given by,

$$
\Omega=\frac{m_{\chi} s_{0} Y_{\chi}\left(x_{0}\right)}{\rho_{c}}
$$

where $s_{0}=2890 \mathrm{~cm}^{-3}$ is the present entropy density and $\rho_{c}=h^{2} 1.9 \times 10^{-29} \mathrm{gm} / \mathrm{cm}^{3}$ is the critical density. We find that by taking $g_{H}=0.087, \delta \sim 10^{-3}$ and $\gamma \sim 10^{-4}$ in eq. (3.7), we obtain the correct relic density $\Omega h^{2}=0.1199 \pm 0.0027$, consistent with Planck [29] and WMAP [30] data. From $g_{H}$ and $\gamma$ we can fix $M_{\theta_{3}} \simeq 1400 \mathrm{GeV}$ and $m_{\chi} \simeq \frac{1}{2} M_{\theta_{3}} \simeq 700 \mathrm{GeV}$. There is a large hierarchy between the fourth generation charged fermion mass and the other charged leptons masses. We do not have any theory for the Yukawa couplings and we take the $m_{\mu^{\prime}}$ mass which fits best the AMS-02 positron spectrum and muon $(g-2)$. A bench mark set of values used in this paper for the masses and couplings is given in table 2 .

\subsection{Comparison with AMS-02 and PAMELA data}

The dark matter in the galaxy annihilates into $\mu^{+} \mu^{-}$and the positron excess seen at AMS-02 $[20,21]$ appears from the decay of muon. We use publicly available code PPPC4DMID [38, 39] to compute the positron spectrum $\frac{d N_{e}+}{d E}$ from the decay of $\mu$ pairs for $700 \mathrm{GeV}$ dark matter. We then use the GALPROP code [40, 41] for propagation, in which we take the annihilation rate $\sigma v_{\mu^{+} \mu^{-}}$, and the positron spectrum $\frac{d N_{e}+}{d E}$ as an input to the differential injection rate,

$$
Q_{e^{+}}(E, \vec{r})=\frac{\rho^{2}}{2 m_{\chi}^{2}}\langle\sigma v\rangle_{\mu^{+} \mu^{-}} \frac{d N_{e^{+}}}{d E}
$$




\begin{tabular}{|c|c|}
\hline Parameters & Numerical values \\
\hline$g_{H}$ & 0.087 \\
\hline$y_{h}$ & 0.037 \\
\hline$y_{A}$ & 0.020 \\
\hline$y_{H^{ \pm}}$ & 0.1 \\
\hline$m_{\chi}$ & $700 \mathrm{GeV}$ \\
\hline$m_{\mu^{\prime}}$ & $740 \mathrm{GeV}$ \\
\hline$M_{\theta_{3}}$ & $1400 \mathrm{GeV}$ \\
\hline$M_{\theta^{+}}$ & $1400 \mathrm{GeV}$ \\
\hline$m_{H^{ \pm}}$ & $1700 \mathrm{GeV}$ \\
\hline$m_{h}$ & $125 \mathrm{GeV}$ \\
\hline$m_{A}$ & $150 \mathrm{GeV}$ \\
\hline$\delta$ & $10^{-3}$ \\
\hline$\gamma$ & $10^{-4}$ \\
\hline
\end{tabular}

Table 2. Bench mark set of values used in the model.

where $\rho$ denotes the density of dark matter in the Milky Way halo, which we take to be the NFW profile [42],

$$
\rho_{\mathrm{NFW}}=\rho_{0} \frac{r_{s}}{r}\left(1+\frac{r}{r_{s}}\right)^{-2}, \quad \rho_{0}=0.4 \mathrm{GeV} / \mathrm{cm}^{3}, \quad r_{s}=20 \mathrm{kpc}
$$

In GALPROP code [40, 41], we take the diffusion coefficient $D_{0}=3.6 \times 10^{28} \mathrm{~cm}^{2} \mathrm{~s}^{-1}$ and Alfven speed $v_{A}=15 \mathrm{Km} \mathrm{s}^{-1}$. We choose, $z_{h}=4 \mathrm{kpc}$ and $r_{\max }=20 \mathrm{kpc}$, which are the half-width and maximum size for 2D galactic model respectively. We choose the nucleus spectral index breaks at $9 \mathrm{GeV}$ and spectral index above this is 2.36 and below is 1.82 . The normalization flux of electron at $100 \mathrm{GeV}$ is $1.25 \times 10^{-8} \mathrm{~cm}^{-2} \mathrm{~s}^{-1} \mathrm{sr}^{-1} \mathrm{GeV}^{-1}$ and for the case of electron, we take breaking point at $4 \mathrm{GeV}$ and its injection spectral index above $4 \mathrm{GeV}$ is $\gamma_{1}^{\text {el }}=2.44$ and below $\gamma_{0}^{\text {el }}=1.6$. After solving the propagation equation, GALPROP [40, 41] gives the desired positron flux.

To fit the AMS-02 data, the input annihilation CS required in GALPROP is, $\sigma v_{\chi \chi \rightarrow \mu^{+} \mu^{-}}=2.33 \times 10^{-25} \mathrm{~cm}^{3} \mathrm{~s}^{-1}$. The annihilation CS for $\mu$ final state from eq. (3.1) is, $\sigma v \approx 2.8 \times 10^{-25} \mathrm{~cm}^{3} \mathrm{~s}^{-1}$, which signifies that there is no extra "astrophysical" boost factor needed to satisfy AMS-02 data. The annihilation rate required for relic density was $\langle\sigma v\rangle \sim 3 \times 10^{-26} \mathrm{~cm}^{3} / \mathrm{sec}$ and the factor $\sim 10$ increase in $\sigma v$ at the present epoch is due to resonant enhancement by taking $m_{\chi} \simeq \frac{1}{2} M_{\theta_{3}}$. In figure 2 , we plot the output of GALPROP code and compare it with the observed AMS-02 [20, 21] and PAMELA [22] data. We see that our positron spectrum fits the AMS-02 data [20, 21] very well. We also check the photon production from the decay of $\mu$ final state by generating the $\gamma$-ray spectrum called $\frac{d N_{\gamma}}{d E}$ from publicly available code PPPC4DMID [38, 39] and propagating it through the GALPROP code $[40,41]$. We then compare the output with the observed Fermi-LAT data [43], as shown in figure 3 , and find that the $\gamma$-ray does not exceed the observed limits. 


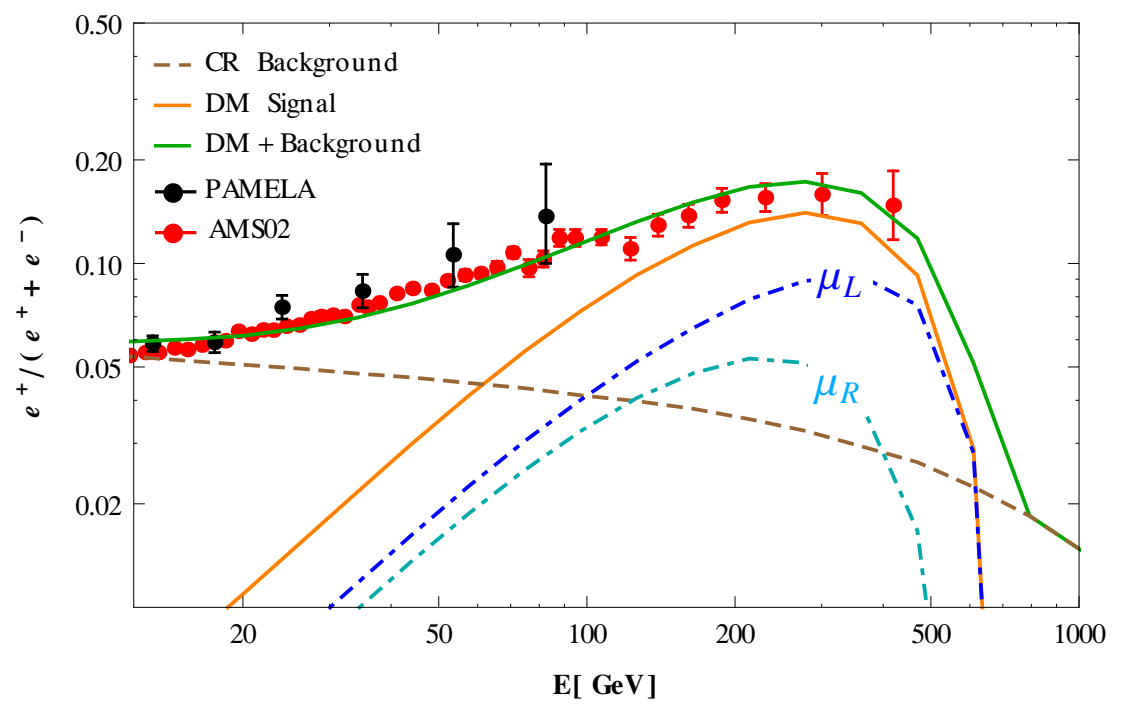

Figure 2. The positron flux spectrum compared with data from AMS-02 [20, 21] and PAMELA [22]. The contributions of different channels $\left(\mu_{L}, \mu_{R}\right)$ are shown for comparison.

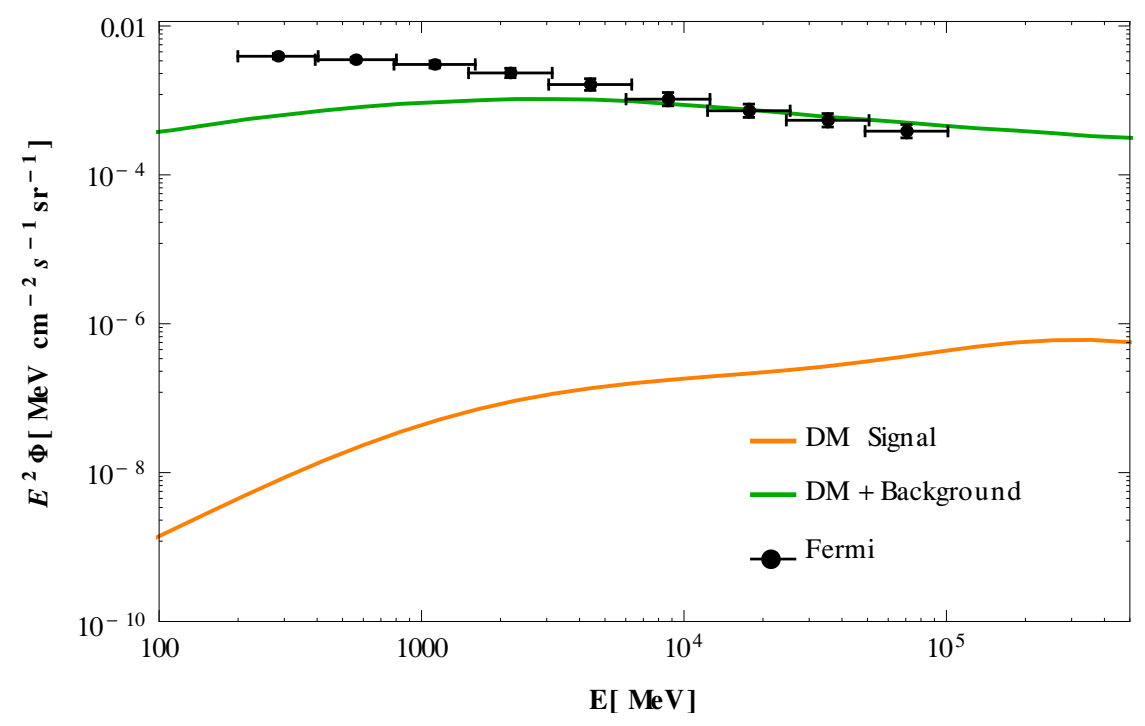

Figure 3. The $\gamma$-ray spectrum compared with data from Fermi Lat [43].

There is no annihilation to hadrons, so no excess of antiprotons are predicted, consistent with the PAMELA [25] and AMS-02 [26] data.

\section{Muon magnetic moment}

The $\mathrm{SU}(2)_{\mathrm{HV}}$ horizontal symmetry, which connects muon and 4th generation families, gives extra contributions to muon $(g-2)$. The diagrams that contribute to muon $(g-2)$ with $\mathrm{SU}(2)_{\mathrm{HV}}$ charged gauge boson $\theta^{+}$and scalar $\eta_{i \alpha}^{\beta}$ are shown in figure 4 . 

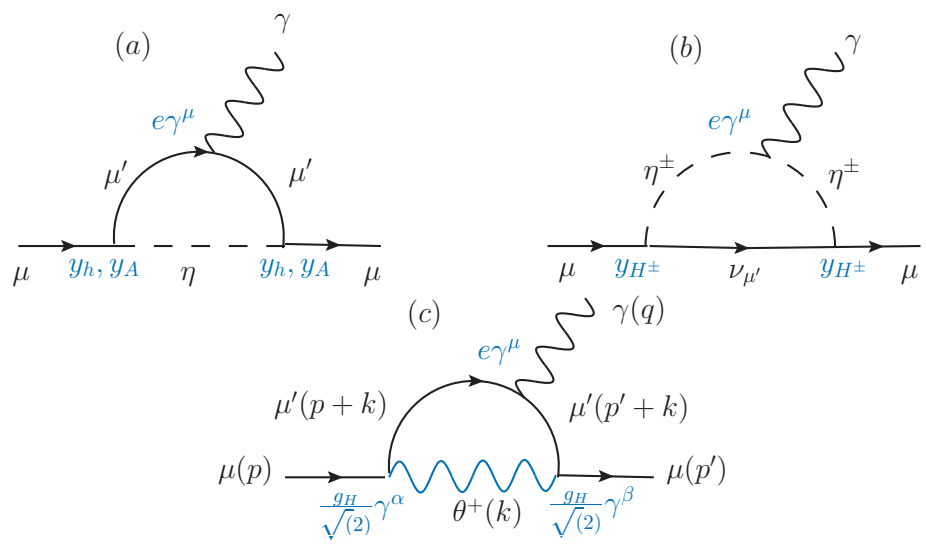

Figure 4. Feynman diagrams of scalar $\eta_{i \alpha}^{\beta}$ and $\mathrm{SU}(2)_{\mathrm{HV}}$ gauge boson $\theta^{+}$, which give contributions to muon $(g-2)$.

We first calculate the contribution from $\mathrm{SU}(2)_{\mathrm{HV}}$ gauge boson $\theta^{+}$, which is shown in figure 4(c). For this diagram the vertex factor of the amplitude $\mu\left(p^{\prime}\right) \Gamma_{\mu} \mu(p) \epsilon^{\mu}$ is,

$$
\Gamma_{\mu}=\frac{e g_{H}^{2}}{2} \int \frac{d^{4} k}{(2 \pi)^{4}} \gamma^{\beta} \frac{\left(\not p^{\prime}+\not k+m_{\mu^{\prime}}\right)}{\left(p^{\prime}+k\right)^{2}-m_{\mu^{\prime}}^{2}} \gamma_{\mu} \frac{\left(\not p+\not k+m_{\mu^{\prime}}\right)}{(p+k)^{2}-m_{\mu^{\prime}}^{2}} \gamma^{\alpha} \frac{g_{\alpha \beta}}{k^{2}-M_{\theta^{+}}^{2}}
$$

we perform the integration and use the Gorden identity to replace,

$$
\left(p_{\mu}+p_{\mu}^{\prime}\right)=2 m_{\mu} \gamma_{\mu}+i \sigma^{\mu \nu} q_{\nu}
$$

and identify the coefficient of the $i \sigma^{\mu \nu} q_{\nu}$ as the magnetic form factor. The contribution to $\Delta a_{\mu}$ is,

$$
\left[\Delta a_{\mu}\right]_{\theta^{+}}=\frac{m_{\mu}^{2}}{16 \pi^{2}} \int_{0}^{1} d x \frac{g_{H}^{2}\left(\frac{2 m_{\mu}^{\prime}}{m_{\mu}}\left(x-x^{2}\right)-\left(x-x^{3}\right)\right)}{(1-x) m_{\mu^{\prime}}^{2}-x(1-x) m_{\mu}^{2}+x M_{\theta^{ \pm}}^{2}}
$$

In the limit of $M_{\theta^{+}}^{2} \gg m_{\mu^{\prime}}^{2}$, we get the anomalous magnetic moment,

$$
\left[\Delta a_{\mu}\right]_{\theta^{+}}=\frac{g_{H}^{2}}{8 \pi^{2}}\left(\frac{m_{\mu} m_{\mu^{\prime}}-2 / 3 m_{\mu}^{2}}{M_{\theta^{+}}^{2}}\right)
$$

we note that in eq. (4.4), the first term is dominant which shows $m_{\mu} m_{\mu^{\prime}}$ enhancement in the muon $(g-2)$.

In our model, the contribution from the neutral higgs $\eta(\mathrm{CP}$-even $h$ and $\mathrm{CP}$-odd $A$ ) is shown in figure $4(\mathrm{a})$. The $(g-2)$ contribution of this diagram is [44],

$$
\begin{aligned}
{\left[\Delta a_{\mu}\right]_{h, A}=} & \frac{m_{\mu}^{2}}{8 \pi^{2}} \int_{0}^{1} d x \frac{y_{h}^{2}\left(x^{2}-x^{3}+\frac{m_{\mu^{\prime}}}{m_{\mu}} x^{2}\right)}{m_{\mu}^{2} x^{2}+\left(m_{\mu^{\prime}}^{2}-m_{\mu}^{2}\right) x+m_{h}^{2}(1-x)} \\
& +\frac{m_{\mu}^{2}}{8 \pi^{2}} \int_{0}^{1} d x \frac{y_{A}^{2}\left(x^{2}-x^{3}-\frac{m_{\mu^{\prime}}}{m_{\mu}} x^{2}\right)}{m_{\mu}^{2} x^{2}+\left(m_{\mu^{\prime}}^{2}-m_{\mu}^{2}\right) x+m_{A}^{2}(1-x)}
\end{aligned}
$$


where $y_{h}, y_{A}$ represent the Yukawa couplings of neutral CP-even and odd higgs respectively and their masses are denoted by $m_{h}$ and $m_{A}$ respectively. We shall calculate the contributions from the lightest scalars only, which give the larger contributions in compare to heavy scalars. In the limits $m_{\mu^{\prime}}^{2} \gg m_{h}^{2}, m_{\mu^{\prime}}^{2} \gg m_{A}^{2}$, doing the integration in eq. (4.5) we get the anomalous magnetic moment,

$$
\left[\Delta a_{\mu}\right]_{h, A}=\frac{1}{8 \pi^{2}}\left(\frac{3 m_{\mu} m_{\mu^{\prime}}\left(y_{h}^{2}-y_{A}^{2}\right)+m_{\mu}^{2}\left(y_{h}^{2}+y_{A}^{2}\right)}{6 m_{\mu^{\prime}}^{2}}\right)
$$

In a similar way, the contribution from the mass eigenstate $H^{ \pm}$of charged higgs $\eta^{ \pm}$, shown in figure $4(\mathrm{~b})$, is given by [44],

$$
\left[\Delta a_{\mu}\right]_{H^{ \pm}}=\frac{m_{\mu}^{2}}{8 \pi^{2}} \int_{0}^{1} d x \frac{y_{H^{ \pm}}^{2}\left(x^{3}-x^{2}+\frac{m_{\nu_{\mu^{\prime}}}}{m_{\mu}}\left(x^{2}-x\right)\right)}{m_{\mu}^{2} x^{2}+\left(m_{H^{ \pm}}^{2}-m_{\mu}^{2}\right) x+m_{\nu_{\mu^{\prime}}}^{2}(1-x)}
$$

where $y_{H^{ \pm}}$and $m_{H^{ \pm}}$are the Yukawa coupling and mass of the charged higgs respectively. We perform the integration (eq. (4.7)) in the limit $m_{H^{ \pm}}^{2} \gg m_{\nu_{\mu^{\prime}}}^{2}$, and get the anomalous magnetic moment,

$$
\left[\Delta a_{\mu}\right]_{H^{ \pm}}=-\frac{y_{H^{ \pm}}^{2}}{8 \pi^{2}}\left(\frac{3 m_{\mu} m_{\nu_{\mu^{\prime}}}+m_{\mu}^{2}}{6 m_{H^{ \pm}}^{2}}\right)
$$

So the complete contribution to muon $(g-2)$ in our model is given as,

$$
\Delta a_{\mu}=\left[\Delta a_{\mu}\right]_{\theta^{+}}+\left[\Delta a_{\mu}\right]_{h, A}+\left[\Delta a_{\mu}\right]_{H^{ \pm}}
$$

As discussed before, in our model the lightest CP-even scalar $h_{1}$ is mainly composed of $\eta$, so we can write,

$$
y_{h} \sim k_{2} \cos \alpha_{1}
$$

where $\alpha_{1}$ is the mixing angle between CP-even mass eigenstate $h_{1}$ and gauge eigenstate $\eta$, and $k_{2}$ is the Yukawa coupling defined in eq. (2.8). In the similar way, we assume that lightest pseudoscalar $A$ and charged higgs $H^{ \pm}$are also mainly composed of $\eta$, so that we can write

$$
y_{A} \sim k_{2} \cos \alpha_{2}, \quad y_{H^{ \pm}} \sim \tilde{k}_{2} \cos \alpha_{3}
$$

where $\alpha_{2}$ is the mixing angle between CP-odd scalars and $\alpha_{3}$ is the mixing angle between the charged scalars. $\tilde{k}_{2}$ denotes the Yukawa coupling defined in eq. (2.8).

In the $\mathrm{SU}(2)_{H}$ gauge boson sector, we take $g_{H}=0.087, M_{\theta^{+}} \approx 1400 \mathrm{GeV}\left(M_{\theta_{3}} \approx M_{\theta^{+}}\right)$, which are fixed from the requirement of correct relic density and we take $m_{\mu^{\prime}}=740 \mathrm{GeV}$, coming from the stability requirement of dark matter $\left(m_{\mu^{\prime}}>m_{\chi}\right)$. After doing numerical calculation, we get $[\Delta a]_{\theta^{+}}=3.61 \times 10^{-9}$.

The contribution from $(h, A)$ scalars depend on the parameter $k_{2}^{2}\left(\cos ^{2} \alpha_{1}-\cos ^{2} \alpha_{2}\right)$, which we assume to be $\simeq 10^{-3}$ and obtain $\left[\Delta a_{\mu}\right]_{h, A}=0.82 \times 10^{-9}$. For the charged scalar contribution, we assume $\tilde{k}_{2} \cos \alpha_{3}=0.1$ and $m_{H^{ \pm}}=1700 \mathrm{GeV}$ and obtain $\left[\Delta a_{\mu}\right]_{H^{ \pm}}=$ $-1.53 \times 10^{-9}$. Adding the contributions from $\theta^{+},(h, A)$ and $H^{ \pm}$, we get

$$
\Delta a_{\mu}=2.9 \times 10^{-9}
$$


which is in agreement with the experimental result $[1,2]$ within $1 \sigma$. To get the desired value of muon $(g-2)$, we have to consider a large hierarchy between the neutral higgs $\left(m_{h} \sim 125 \mathrm{GeV}, m_{A} \sim 150 \mathrm{GeV}\right)$ and the charged higgs $m_{H^{ \pm}} \sim 1700 \mathrm{GeV}$. These masses have to arise by appropriate choices of the couplings in the higgs potential of $\left(\phi_{i}, \eta_{i \alpha}^{\beta}, \chi_{\alpha}\right)$.

\section{Result and discussion}

We studied a 4th generation extension of the standard model, where the 4th generation leptons interact with the muon family via $\mathrm{SU}(2)_{\mathrm{HV}}$ gauge bosons. The 4 th generation righthanded neutrino is identified as the dark matter. We proposed a common explanation to the excess of positron seen at AMS-02 [20, 21] and the discrepancy between SM prediction [3$9]$ and BNL measurement $[1,2]$ of muon $(g-2)$. The $\mathrm{SU}(2) \mathrm{HV}$ gauge boson $\theta^{+}$with 4th generation charged lepton $\mu^{\prime}$ and charged higgs $H^{ \pm}$, give the required contribution to muon $(g-2)$ to satisfy the BNL measurement $[1,2]$ within $1 \sigma$. The LHC constraints on 4 th generation quarks is evaded by extending the higgs sector as in $[35,36]$. In our horizontal $\mathrm{SU}(2)_{\mathrm{HV}}$ gauge symmetry model, we also explain the preferential annihilation of dark matter to $\mu^{+} \mu^{-}$channel over other leptons and predict that there is no antiproton excess, in agreement with PAMELA [25] and AMS-02 [26] data. Since the dark matter has gauge interactions only with the muon family at tree level, we can evade the bounds from direct detection experiments $[45,46]$ based on scattering of dark matter with the first generation quarks.

Open Access. This article is distributed under the terms of the Creative Commons Attribution License (CC-BY 4.0), which permits any use, distribution and reproduction in any medium, provided the original author(s) and source are credited.

\section{References}

[1] Muon G-2 collaboration, G.W. Bennett et al., Final report of the E821 muon anomalous magnetic moment measurement at BNL, Phys. Rev. D 73 (2006) 072003 [hep-ex/0602035] [INSPIRE].

[2] Muon (G-2) collaboration, G.W. Bennett et al., An improved limit on the muon electric dipole moment, Phys. Rev. D 80 (2009) 052008 [arXiv:0811.1207] [INSPIRE].

[3] T. Aoyama, M. Hayakawa, T. Kinoshita and M. Nio, Complete tenth-order QED contribution to the muon g-2, Phys. Rev. Lett. 109 (2012) 111808 [arXiv:1205.5370] [INSPIRE].

[4] C. Gnendiger, D. Stöckinger and H. Stöckinger-Kim, The electroweak contributions to $(g-2)_{\mu}$ after the Higgs boson mass measurement, Phys. Rev. D 88 (2013) 053005 [arXiv: 1306.5546] [INSPIRE].

[5] M. Davier, A. Hoecker, B. Malaescu and Z. Zhang, Reevaluation of the hadronic contributions to the muon $g-2$ and to $\alpha\left(M_{Z}^{2}\right)$, Eur. Phys. J. C 71 (2011) 1515 [Erratum ibid. C 72 (2012) 1874] [arXiv:1010.4180] [INSPIRE].

[6] K. Hagiwara, R. Liao, A.D. Martin, D. Nomura and T. Teubner, $(g-2)_{\mu}$ and $\alpha\left(M_{Z}^{2}\right)$ re-evaluated using new precise data, J. Phys. G 38 (2011) 085003 [arXiv:1105.3149] [INSPIRE]. 
[7] M. Benayoun, P. David, L. DelBuono and F. Jegerlehner, An update of the HLS estimate of the muon $g-2$, Eur. Phys. J. C 73 (2013) 2453 [arXiv:1210.7184] [InSPIRE].

[8] T. Blum et al., The muon $(g-2)$ theory value: present and future, arXiv:1311.2198 [INSPIRE].

[9] J.P. Miller, E. de Rafael, B.L. Roberts and D. Stöckinger, Muon $(g-2)$ : experiment and theory, Ann. Rev. Nucl. Part. Sci. 62 (2012) 237 [INSPIRE].

[10] Particle Data Group collaboration, J. Beringer et al., Review of particle physics, Phys. Rev. D 86 (2012) 010001 [INSPIRE].

[11] T. Moroi, The Muon anomalous magnetic dipole moment in the minimal supersymmetric standard model, Phys. Rev. D 53 (1996) 6565 [Erratum ibid. D 56 (1997) 4424] [hep-ph/9512396] [INSPIRE].

[12] D. Stöckinger, The muon magnetic moment and supersymmetry, J. Phys. G 34 (2007) R45 [hep-ph/0609168] [INSPIRE].

[13] S. Baek, N.G. Deshpande, X.G. He and P. Ko, Muon anomalous $g-2$ and gauged $L_{\mu}-L_{\tau}$ models, Phys. Rev. D 64 (2001) 055006 [hep-ph/0104141] [INSPIRE].

[14] F.S. Queiroz and W. Shepherd, New physics contributions to the muon anomalous magnetic moment: a numerical code, Phys. Rev. D 89 (2014) 095024 [arXiv: 1403.2309] [INSPIRE].

[15] P. Agrawal, Z. Chacko and C.B. Verhaaren, Leptophilic dark matter and the anomalous magnetic moment of the muon, JHEP 08 (2014) 147 [arXiv:1402.7369] [INSPIRE].

[16] Y. Bai and J. Berger, Lepton portal dark matter, JHEP 08 (2014) 153 [arXiv:1402.6696] [INSPIRE].

[17] A. Freitas, J. Lykken, S. Kell and S. Westhoff, Testing the muon $g-2$ anomaly at the LHC, JHEP 05 (2014) 145 [Erratum ibid. 09 (2014) 155] [arXiv:1402.7065] [INSPIRE].

[18] C.D. Carone, Flavor-nonuniversal dark gauge bosons and the muon $g-2$, Phys. Lett. B 721 (2013) 118 [arXiv:1301.2027] [INSPIRE].

[19] S. Bar-Shalom, S. Nandi and A. Soni, Muon $g-2$ and lepton flavor violation in a two Higgs doublets model for the fourth generation, Phys. Lett. B 709 (2012) 207 [arXiv:1112.3661] [INSPIRE].

[20] AMS collaboration, M. Aguilar et al., First result from the Alpha Magnetic Spectrometer on the International Space Station: precision measurement of the positron fraction in primary cosmic rays of 0.5-350 GeV, Phys. Rev. Lett. 110 (2013) 141102 [INSPIRE].

[21] AMS collaboration, L. Accardo et al., High statistics measurement of the positron fraction in primary cosmic rays of 0.5-500 GeV with the Alpha Magnetic Spectrometer on the International Space Station, Phys. Rev. Lett. 113 (2014) 121101 [INSPIRE].

[22] PAMELA collaboration, O. Adriani et al., An anomalous positron abundance in cosmic rays with energies 1.5-100 GeV, Nature 458 (2009) 607 [arXiv:0810.4995] [INSPIRE].

[23] M. Das and S. Mohanty, Leptophilic dark matter in gauged $L_{\mu}-L_{\tau}$ extension of MSSM, Phys. Rev. D 89 (2014) 025004 [arXiv: 1306.4505] [INSPIRE].

[24] A. De Simone, A. Riotto and W. Xue, Interpretation of AMS-02 results: correlations among dark matter signals, JCAP 05 (2013) 003 [arXiv: 1304.1336] [INSPIRE].

[25] PAMELA collaboration, O. Adriani et al., PAMELA results on the cosmic-ray antiproton flux from $60 \mathrm{MeV}$ to $180 \mathrm{GeV}$ in kinetic energy, Phys. Rev. Lett. 105 (2010) 121101 [arXiv: 1007.0821] [INSPIRE].

[26] AMS-02 collaboration, http://www.ams02.org/. 
[27] S. Baek and P. Ko, Phenomenology of $\mathrm{U}(1)_{L_{\mu}-L_{\tau}}$ charged dark matter at PAMELA and colliders, JCAP 10 (2009) 011 [arXiv:0811.1646] [INSPIRE].

[28] P.S.B. Dev, D.K. Ghosh, N. Okada and I. Saha, Neutrino mass and dark matter in light of recent AMS-02 results, Phys. Rev. D 89 (2014) 095001 [arXiv:1307.6204] [INSPIRE].

[29] Planck collaboration, P.A.R. Ade et al., Planck 2013 results. XVI. Cosmological parameters, Astron. Astrophys. 571 (2014) A16 [arXiv:1303.5076] [INSPIRE].

[30] WMAP collaboration, G. Hinshaw et al., Nine-year Wilkinson Microwave Anisotropy Probe (WMAP) observations: cosmological parameter results, Astrophys. J. Suppl. 208 (2013) 19 [arXiv: 1212.5226] [INSPIRE].

[31] M. Ibe, H. Murayama and T.T. Yanagida, Breit-Wigner enhancement of dark matter annihilation, Phys. Rev. D 79 (2009) 095009 [arXiv:0812.0072] [INSPIRE].

[32] W.-L. Guo and Y.-L. Wu, Enhancement of dark matter annihilation via Breit-Wigner resonance, Phys. Rev. D 79 (2009) 055012 [arXiv:0901.1450] [INSPIRE].

[33] X.-J. Bi, X.-G. He and Q. Yuan, Parameters in a class of leptophilic models from PAMELA, ATIC and FERMI, Phys. Lett. B 678 (2009) 168 [arXiv:0903.0122] [INSPIRE].

[34] E. Witten, An SU(2) anomaly, Phys. Lett. B 117 (1982) 324 [inSPIRE].

[35] S. Bar-Shalom, S. Nandi and A. Soni, Two Higgs doublets with 4th generation fermions: models for TeV-scale compositeness, Phys. Rev. D 84 (2011) 053009 [arXiv:1105.6095] [INSPIRE].

[36] X.-G. He and G. Valencia, An extended scalar sector to address the tension between a fourth generation and Higgs searches at the LHC, Phys. Lett. B 707 (2012) 381 [arXiv:1108.0222] [INSPIRE].

[37] C.L. Ong, A study of a model with horizontal gauge symmetry at one loop level, Phys. Rev. D 22 (1980) 2886 [INSPIRE].

[38] M. Cirelli et al., PPPC \& DM ID: a poor particle physicist cookbook for dark matter indirect detection, JCAP 03 (2011) 051 [Erratum ibid. 10 (2012) E01] [arXiv:1012.4515] [INSPIRE].

[39] P. Ciafaloni et al., Weak corrections are relevant for dark matter indirect detection, JCAP 03 (2011) 019 [arXiv: 1009.0224] [InSPIRE].

[40] A.W. Strong, I.V. Moskalenko and V.S. Ptuskin, Cosmic-ray propagation and interactions in the galaxy, Ann. Rev. Nucl. Part. Sci. 57 (2007) 285 [astro-ph/0701517] [InSPIRE].

[41] I.V. Moskalenko and A.W. Strong, Production and propagation of cosmic ray positrons and electrons, Astrophys. J. 493 (1998) 694 [astro-ph/9710124] [INSPIRE].

[42] J.F. Navarro, C.S. Frenk and S.D.M. White, The structure of cold dark matter halos, Astrophys. J. 462 (1996) 563 [astro-ph/9508025] [INSPIRE].

[43] Fermi-LAT collaboration, A.A. Abdo et al., The spectrum of the isotropic diffuse gamma-ray emission derived from first-year Fermi Large Area Telescope data, Phys. Rev. Lett. 104 (2010) 101101 [arXiv:1002.3603] [INSPIRE].

[44] J.P. Leveille, The second order weak correction to $(g-2)$ of the muon in arbitrary gauge models, Nucl. Phys. B 137 (1978) 63 [inSPIRE].

[45] XENON100 collaboration, E. Aprile et al., Dark matter results from 225 live days of XENON100 data, Phys. Rev. Lett. 109 (2012) 181301 [arXiv:1207.5988] [INSPIRE].

[46] LUX collaboration, D.S. Akerib et al., First results from the LUX dark matter experiment at the Sanford Underground Research Facility, Phys. Rev. Lett. 112 (2014) 091303 [arXiv:1310.8214] [INSPIRE]. 